\title{
Incertidumbre, inestabilidad y complejidad
}

\author{
Marcelo Manucci \\ Pontificia Universidad Javeriana (Colombia) \\ marcelomanucci@gmail.com \\ Recepción: 14/05/2021 \\ Aceptación:15/06/21
}

\section{Introducción}

Los primeros años de este siglo han sido uno de los momentos históricos más paradojales que el mundo haya vivido en las últimas décadas con un vasto paisaje de procesos contradictorios, movimientos impredecibles y situaciones inéditas que irrumpieron más allá de las previsiones y los pronósticos, inclusive antes de la pandemia de la COVID-19. La última debacle financiera mundial del 2008 no solo ha dejado las heridas de una estructura económica deliberadamente idealizada, sino también y más profundamente, las fisuras de un modelo de realidad basado en certezas ideológicamente forzadas y técnicamente aventuradas. El colapso financiero del final de la primera década del siglo (que arrasó con ideales económicos, mitos financieros, gobiernos, historias y fantasías de crecimiento) no fue más que un síntoma de una forma imperante de ver y actuar en el mundo. Quizá uno de los síntomas más escandalosos por su dimensión ética y política, pero no fue el único. El final de las certezas al cual asistimos en estos últimos años no es más que el final de una cosmovisión imperante y hegemónica de ordenar e intervenir en la realidad. La pintura idílica de un mundo ordenado y previsible se ha trasformado en una dinámica desbocada de consecuencias inciertas que se agravaron con la pandemia de la COVID-19.

Probablemente estamos viviendo la misma incertidumbre de aquel hombre medieval que miraba absorto su mundo cotidiano que había dejado de ser una prolija trama de explicaciones aristotélicas y religiosas para transformarse en un conjunto de incógnitas filosóficas, desafíos tecnológicos y amenazas espirituales. Desde aquellos hombres posmedievales, hemos vivido períodos de desarrollo y grandes avances y beneficios tecnológicos. Pero también ha sido muy alto el costo de esta dinámica de intervención en los procesos sociales y económicos. Los paradigmas que han mantenido un ordenamiento post medieval por cuatrocientos años están mostrando sus fisuras representadas por metodologías, creencias, visiones, actitudes y perspectivas que marcan el desconcierto de nuestros días. Frente a esta cosmovisión aparece un mundo distinto con una dinámica que nos parece caótica porque recién estamos juntando las piezas dispersas de una perspectiva desactualizada. 


\section{Metáforas del desconcierto}

El espacio de intervención de las organizaciones se presenta complejo por naturaleza, inestable e incierto. La mundialización de los intercambios y la competencia, la puesta en redes mundiales de la información, la redefinición de las grandes ideologías estructurantes, la aceleración de los fenómenos migratorios y el debilitamiento de numerosos referentes, entre otros fenómenos, han hecho crecer en muy poco tiempo el grado de complejidad y de incertidumbre de nuestras sociedades.

El abordaje de la dinámica de la realidad se realiza siempre a través de un modelo de esa realidad. Las estrategias de intervención surgen de ese modelo corporativo de realidad. Por lo tanto, si el modelo de realidad funciona, las intervenciones son operativas, la organización puede interactuar fluidamente en su entorno. Cuando el modelo no funciona aparece el desconcierto. Cuando las construcciones no alcanzan para aprehender a la realidad aparece la impotencia y la amenaza. La garantía de credibilidad los modelos se diluyen en los acontecimientos que nos rodean.

Todo modelo de abordaje de la realidad siempre tiene determinadas garantías implícitas o explícitas (metodológicas, científicas, filosóficas, técnicas) que respaldan los postulados y los principios de utilización. Para salir del desconcierto, no solo es necesario redefinir el modelo sino también es necesario confiar en las garantías de ese modelo. Al abordar la realidad, siempre estamos saltando de construcciones en construcciones, de modelos en modelos, por eso de la misma manera como llegamos al desconcierto podemos salir, con un nuevo modelo, con nuevas construcciones, con las mismas o diferentes garantías.

El desconcierto pareciera ser la herencia de la segunda mitad del siglo pasado a nuestros días. Pero la presencia del desconcierto es mucho más profunda. La historia del desconcierto es la historia del hombre creando modelos de abordaje, con diferentes garantías, para tratar de entender e intervenir sobre su entorno. Las ciencias han tenido muchos momentos de desconcierto, luego de los cuales surgían nuevas miradas y modelos de realidad. Las metáforas del desconcierto son las metáforas de los hombres saltando de construcción en construcción, de formulaciones en formulaciones, de garantías en garantías. A pesar de los siglos y las culturas Galileo, Newton, Einsten, Heisenberg, Schroedinger, Prigogine y otros hombres de ciencia hasta la actualidad tienen muchos aspectos en común, todos se enfrentaron al desconcierto de sus propios modelos teóricos al tratar de entender determinados fenómenos de la realidad. Muchos de los conceptos que conforman la cosmovisión y la metodología de las organizaciones de hoy son el legado del desconcierto de estos hombres a lo largo de la historia.

\section{Los límites de nuestra realidad}

En el nivel individual, las hormigas despliegan un amplio repertorio de capacidades cognitivas y computacionales. Cada hormiga es capaz de percibir una gran cantidad de entradas sensoriales, modular su comportamiento de acuerdo con múltiples estímulos y tomar decisiones con base en grandes cantidades de información. Se conoce, por ejemplo, que las hormigas pueden distinguir entre cientos de químicos distintos (Gordon, 2016a). Con su diminuto cerebro, que pesa alrededor de 0.1 miligramos (Ronacher, 2008), las hormigas aprenden, planean para el futuro, enseñan, toman decisiones sofisticadas y emplean herramientas (Dornhaus \& Franks, 2008). El uso de herramientas, particularmente, se ha detectado en el transporte de líquidos y en la construcción de nidos. La figura 1 (arriba) muestra a una hormiga tejedora australiana adulta (Oecophylla) sosteniendo una larva entre sus mandíbulas y golpeándola suavemente con sus antenas como indicación para que comience a secretar seda de sus glándulas salivares (National Geographic, 2011). La seda es empleada para unir las hojas que formarán parte del nido (abajo).

Werner Heinsenberg, el físico que definió matemáticamente a la incertidumbre, solía decir que "en cada acto de observación se pierde más información de la que se obtiene". Mirar la realidad implica un intricado laberinto donde los fenómenos se entrecruzan con ideas, experiencias, expectativas, emociones y actitudes. Desde este abigarrado tapiz se define un espacio de acción en el presente y se proyecta un horizonte de resultados en el futuro. Resulta interesante pensar, antes de actuar, ¿hacia dónde estamos mirando?, para reflexionar acerca de la posición desde donde se ordenan los fragmentos de realidad que luego se traducen en estrategia, acciones y resultados en las organizaciones.

Mirar está relacionado directamente con la percepción del mundo. En definitiva, al mirar la realidad la estamos construyendo. En el mismo acto miramos, relacionamos y construimos sentido. Por ello, más información no implica mayor claridad. La información recolectada no es aséptica; todo depende de la red de significados desde donde se mira la realidad. Mirar es construir, mirar es interpretar. Mirar en la incertidumbre es como mirarse en un espejo fragmentado, nunca tenemos la imagen completa de la realidad. No hay una certeza objetiva en la configuración de la realidad. 
El físico John Archibald Wheeler planteaba la idea que "el universo es un acto de observación". El universo es participativo porque sin nuestra intervención no tiene sentido hablar de realidad. Nuestro papel como observadores es hacer emerger una definición de la realidad. Por lo tanto, la realidad es lo que las personas perciben como tal. En la misma línea de pensamiento, Danah Zohar plantea que lo que sucede en la realidad depende de cómo la vemos, esto implica que nuestra mera interferencia en la naturaleza, la transforma. Este postulado resalta el papel esencial de la observación, del diálogo entre diversos factores de la realidad y el observador en la formación de realidad. Abner Shimony sostiene que la realidad es una trama de posibilidades que encuentran su expresión material en un punto de decisión o suceso, donde diferentes posibilidades se actualizan y generan un nuevo estado. Cuando se alcanza ese estado, la realidad se torna tan objetiva como la estudiada por las ciencias clásicas. Ilya Prigogine resume en la siguiente frase un nuevo rol del observador en la definición de nuestra vida cotidiana: "Sea lo que fuere lo que llamamos realidad, sólo se nos revela a través de una construcción activa de la cual participamos."

El universo de interrelaciones conlleva una enorme cuota de riesgo. Lo que sucede generalmente es que se busca controlar el riesgo desde un paradigma de simplicidad. Muchas de las soluciones de gestión basadas exclusivamente en los resultados tienen esta lógica: la búsqueda de las causas iniciales para sofocarlas. Esta visión simplista de las relaciones y los comportamientos generalmente conlleva a acciones estereotipadas y mutiladas que encierran a la organización en una cápsula tan cómoda como vulnerable.

Considerar la incertidumbre como espacio de intervención tiene un carácter metodológico. Permite diferenciar la complejidad del entorno como una propiedad de los sistemas sociales, de la percepción de los espacios en los cuales concretamente la organización realiza intervenciones. La interacción cotidiana genera incertidumbre. Las intervenciones que la organización define en su medioambiente interactúan con otras variables y surgen nuevos sucesos. Algunos de esos eventos se pueden prever, en función de experiencias pasadas, pero otros tantos quedan fuera de un horizonte predictivo.

La dinámica compleja del contexto es diferente del espacio de intervención que define una persona (individual o colectivamente). El territorio que definimos "como realidad" es un espacio recortado por la mirada; por lo tanto, es limitado. Por lo tanto, lo que denominamos "la realidad" y que compartimos colectivamente, es un espacio enmarcado por un horizonte predictivo que define un territorio de acción. La incertidumbre está en nuestra propia mirada sobre una dinámica compleja imposible de enlazar y controlar con paradigmas y metodologías.

\section{Desafiar a las certezas}

Más allá de lo que hoy se puede ver como paisaje actual de desarrollo existen posibilidades que quedan ahogadas si manejamos nuestras intervenciones desde la certeza. Trascender las certezas implica desafiar los límites de la mirada respecto del entorno, diseñar alternativas de acción y crear realidades compartidas que posibiliten el crecimiento de las personas comprometidas con los destinos de la organización. Trascender los límites de nuestro horizonte es un desafío a las creencias, los modelos mentales y los hábitos con los que definimos nuestras propias posibilidades y territorios de desarrollo.

En este sentido, la distinción entre complejidad e incertidumbre marca una diferencia entre la dinámica de la realidad que es la complejidad del contexto en el que vive la organización) y la realidad subjetiva que es una construcción, lo que la una persona, grupo u organización percibe e interpreta y define como realidad. La dinámica de la realidad implica un conjunto de variables que en su interacción generan situaciones emergentes que trascienden los límites de la mirada subjetiva. La dinámica compleja de esta trama es difícil de someter a un control absoluto a lo largo del tiempo. La realidad subjetiva es la construcción (individual o compartida) dentro de la cual vivimos y define un territorio de acción, el margen de movimientos. En este marco, la incertidumbre es inherente a cualquier proyecto porque, como toda construcción, tiene los límites de un modelo.

Pensar la realidad como construcción cambia la concepción del espacio y amplía las posibilidades de intervención. Trabajando sobre las percepciones que enmarcan la construcción subjetiva de la realidad, se redefinen aspectos de esa figura subjetiva, al cambiar la percepción subjetiva de la realidad se modifica la gestión de las acciones sobre el entorno.

Las personas somos constructores de sentido, creamos nuestro propio mapa de navegación sobre un mundo fragmentado y dinámico. Nuestro modelo de realidad es la realidad que vivimos. Moldeando las percepciones se pueden crear nuevas realidades. Las percepciones fragmentadas y el modelo global de realidad interactúan inseparablemente. El modelo se nutre de los fragmentos y al mismo tiempo brinda una unidad que da sentido y coherencia a las percepciones cotidianas. Cuando cambia el modelo de la realidad, cambia el sen- 
tido de muchos fenómenos cotidianos y se redefinen las percepciones y las intervenciones. Al mismo tiempo, los resultados de las intervenciones influyen en las percepciones y en el modelo que da sentido a la realidad corporativa.

Acceder a la complejidad a través de la creación de modelos es la metodología que el hombre ha utilizado a lo largo de su vida para interpretar, comprender y prever los movimientos del entorno. En este sentido, uno de los factores esenciales que aparecen ligados a los modelos son los resultados. Cuando a través de la utilización de determinados modelos se obtienen resultados esperados estos modelos pasan a formar parte del hábito cotidiano. Estos modos de percibir, interpretar y prever se naturalizan. De esta manera, la complejidad encuentra un orden momentáneo.

Nuestra estructura humana necesita de cierta estabilidad en su entorno para poder moverse. Así, cuando un modelo alcanza los resultados deseados aparece una ilusión de equilibrio que permite transitar y mantenerse en la dinámica de la realidad. Cuando nuestros modelos sucumben a la dinámica de realidad aparece el desconcierto.

Muchas veces asociamos la incertidumbre con el desconcierto y la cargamos de impotencia. La incertidumbre es el límite de la predicción de nuestros modelos. El desconcierto es el límite de la operatividad cuando la realidad supera lo que nuestros modelos, nuestros paradigmas, nuestras metodologías pueden integrar.

\section{Conclusión}

Reconocer que la incertidumbre está en nuestra propia mirada de la complejidad abre a un universo de posibilidades, simplemente porque más allá de lo que hoy estamos viendo hay un mapa de alternativas por explorar. La potencialidad de la incertidumbre, surge con el cambio de escala de análisis de la realidad. Cuando nuestra mirada se abre a un universo diferente de problemáticas, de las certezas a las probabilidades; del mecanismo cerrado a los sistemas dinámicos. Desde una concepción mecanicista, la incertidumbre aparece como un espacio amenazante, imposible de reducir con palabras claves o ideas totalizadoras. Así, cuando se piensa en una realidad compleja, el sinónimo clásico es confusión, falta de claridad. En la concepción cotidiana, es complejo aquello que no puede retrotraerse a una ley o no se puede reducir a una idea simple.

Las personas, tanto en lo individual como grupal organizamos la visión del mundo desde determinados modelos, como principios rectores, que facilitan las intervenciones en la realidad. Avanzar en este territorio de límites difusos y comportamientos inciertos, implica la necesidad de un pensamiento complejo. Un pensamiento multidimensional, que pueda enfrentar el entramado de las relaciones, el azar y la paradoja de lo uno y múltiple.

\section{Bibliografía}

Ackoff, R.; Gharajedaghi, J. "Mechanics, Organisms and Social Systems", en Strategic Management Journal. Vol. 5, 1984.

Benko, Cathleen. McFarlan, Warren. Connecting the dots. aligning projects with objectives in unpredictable times. Harvard Business School Press. Boston, Massachusetts, 2003.

Corner, Daryl L. Leading at the edge of chaos. John Wiley \& Sons INC. 1998.

Gharajedaghi, J. Systems Methodology. A Holistic Language of Interaction And Design Seeing Through Chaos and Understanding Complexities. Ackoff Center for Advancement of Systems Approaches. University of Pennsylvania. 2004.

Halpern, Joseph Y. Reasoning about Uncertainty. The MIT Press. Cambridge, Massachusetts. London, England. 2003 Massachusetts Institute of Technology.

Lucas, Chris. The Spirit of Complexity. CALResCo Group (Complexity \& Artificial Life Research), Manchester U.K. (Dynamical Psychology Journal January 1999).

Reeves, Hubert. "Incursión en el mundo acausal", en: Reeves, Priban y otros. La Sincronicidad. ¿Existe un orden a-causal? Gedisa. Barcelona, 1987.

Reeves, Priban y otros. La Sincronicidad. ¿Existe un orden a-causal? Gedisa. Barcelona, 1987.

Youngblood, Mark D. "Leadership at the edge of chaos: from control to creativity", en Strategy \& Leadership. Sept-Oct. 1997 v25 n5.

Zohar, Danah. O ser quântico. Una visão revolucionária da naturaza humana e da conciência, baseada na nova física. Editora Best Seller (130 edição). San Pablo, 2000. 